\title{
Extended disease-free interval of 6 years in a recurrent glioblastoma multiforme patient treated with G207 oncolytic viral therapy
}

This article was published in the following Dove Press journal:

Oncolytic Virotherapy

30 January 2015

Number of times this article has been viewed

\section{Thomas R Whisenhunt Jr Kiran F Rajneesh James R Hackney James M Markert}

Department of Neurosurgery, University of Alabama at Birmingham, Birmingham, AL, USA
Correspondence: James M Markert 510 20th Street South, Birmingham, AL 35294-34I0, USA

Tel + I 2059347 7 I I

Email jmarkert@uabmc.edu
Background: Glioblastoma multiforme $(\mathrm{GBM})$ is a relentless primary central nervous system malignancy that remains resistant to conventional therapy despite major advances in clinical neurooncology. This report details the case of a patient who had failed conventional treatment for recurrent GBM and was ultimately treated with a genetically engineered herpes simplex virus (HSV) type 1 vector, G207.

Methods: Case report detailing the outcomes of one patient enrolled into the gene therapy arm of the Neurovir G207 protocol whereby stereotactic injection of $120 \mu \mathrm{L}$ G207 viral suspension containing $1 \times 10^{7}$ plaque-forming units (or active viral particles) was made into the enhancing region of the tumor.

Results: In this patient, despite aggressive surgical resection, adjuvant radiotherapy and chemotherapy, tumor progression occurred. However, with G207 oncolytic therapy and brief exposures to second and third treatments, this patient had an extended survival time of 7.5 years and a 6-year apparent disease-free interval, an extraordinarily unusual finding in the pretemozolomide era.

Conclusion: With minimal adjunctive chemotherapy, including one course of temozolomide, one course of procarbazine, and four cycles of irinotecan, the patient survived over 7 years before the next recurrence. Addition of G207 to this patient's traditional therapy may have been the critical treatment producing her prolonged survival. This report demonstrates the potential for long-term response to a one-time treatment with oncolytic HSV and encourages continued research on oncolytic viral therapy for GBM.

Keywords: oncolytic virotherapy, malignant glioma, tumor, herpes simplex, HSV-1, immunotherapy

\section{Introduction}

Glioblastoma multiforme (GBM), a subset of malignant glioma, accounts for $60 \%$ of the primary malignant brain tumors in United States. ${ }^{1}$ GBMs, classified according to World Health Organization criteria as grade IV astrocytomas, are primarily composed of poorly differentiated astrocytes, progress rapidly, and carry a grave prognosis. ${ }^{2}$ Despite major advances in neurosurgery, neuroradiology, radiation oncology, neurooncology, and supportive measures in treatment of GBM, overall prognosis has not been significantly altered for these patients in the past two decades. ${ }^{3}$ Recent addition of concurrent temozolomide to standard external beam radiation protocols has increased overall survival only modestly, from 12 to approximately 15 months. ${ }^{4}$

The standard management of GBM involves surgery, radiotherapy, and ultimately chemotherapy. ${ }^{5}$ However, not every patient is a candidate for standard management due to age, tumor location, and/or comorbid medical conditions. Standard management 
with aggressive surgical resections, high dose radiation, and chemotherapeutics exposes patients to high risks, with only limited benefit. Due to the overall short survival of patients with GBM, particularly those without methylation of the MGMT promoter, ${ }^{6}$ further advances in the current management modalities as well as consideration of novel biological and immunological therapies is necessary. ${ }^{7-9}$ Among these newer approaches of treatment is a genetically engineered herpes simplex virus (HSV), G207. ${ }^{10-12}$ G207 is engineered with a 1,000 bp deletion of the $\gamma_{1} 34.5$ gene and contains a disabling lacZ insertion in the $U_{L} 39$ locus. These two genes ordinarily circumvent host immune response and allow innate replication of the virus within nondividing cells such as neurons, respectively. ${ }^{13-15}$ The destructive insertion of lac $Z$ within the ribonucleotide reductase gene adds another layer of safety. A two-center (University of Alabama at Birmingham [UAB] and Georgetown University) Phase I clinical trial with G207 was undertaken and has been previously reported. ${ }^{13}$

\section{Patient presentation and management}

A 52-year-old Caucasian female presented with the chief complaint of newly onset focal motor seizures. She had multiple medical conditions including hypertension, type 2 diabetes mellitus, and peripheral vascular disease. Her neurological examination was unremarkable. Magnetic resonance imaging (MRI) revealed a single ring-enhancing lesion in the right frontoparietal region for which the patient only underwent a biopsy. Neuropathologic examination revealed a GBM with an infiltrative glial tumor, with neoplastic astrocytes exhibiting nuclear pleomorphism and hyperchromatosis, along with elements of endothelial proliferation, necrosis, and pseudopalisading architecture (Figure 1A and B). Immunohistochemistry for MIB-1, ${ }^{16}$ a monoclonal antibody against the proliferative antigen Ki67, labeled approximately $33 \%$ of the cells in the biopsy specimen. The patient chose to participate in a Phase III study and was
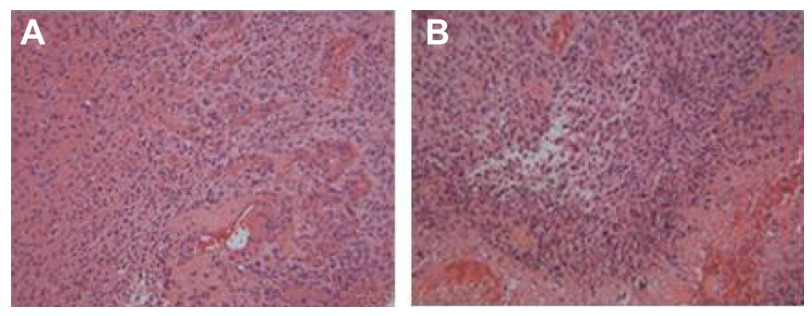

Figure I Low-power photomicrograph of patient's original glioblastoma showing pseudopalisading necrosis (A) and proliferative endothelium (B).

Note: Hematoxylin and eosin stain; $\times 220$. randomized to the standard treatment arm, not receiving any experimental therapy at that time (Figure $2 \mathrm{~A}-\mathrm{F}$ ). The patient underwent craniotomy and resection of the tumor followed by conventional external beam radiotherapy and received a total of $6,000 \mathrm{cGy}$.

Although her neurological examination was unremarkable, she developed bifrontal headaches 3 months after the resection. MRI showed a ring of enhancement in the previous resection site, likely representing tumor progression, radiation changes, and/or postsurgical changes. The entity of pseudoprogression, well recognized in today's era of concurrent temozolomide and radiation therapy, was neither recognized nor common at this time. Findings were felt to be classic for tumor recurrence, and no signs and symptoms of abscess were present, so biopsy was not felt to be indicated. One course of lomustine (CCNU) at $120 \mathrm{mg} / \mathrm{m}^{2}$ dosage, a total dose of $240 \mathrm{mg}$, was administered. Six weeks later, she was revaluated with MRI, which still showed the ring enhancement in

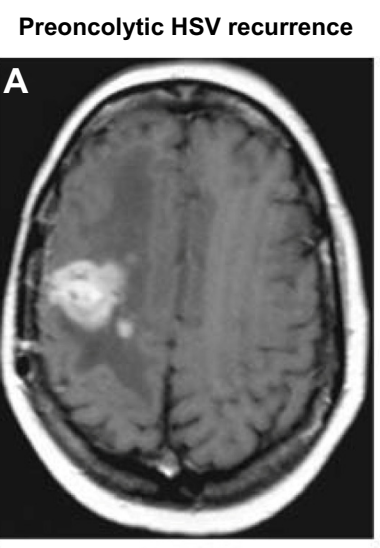

\section{Postoncolytic HSV 5yr F/U}
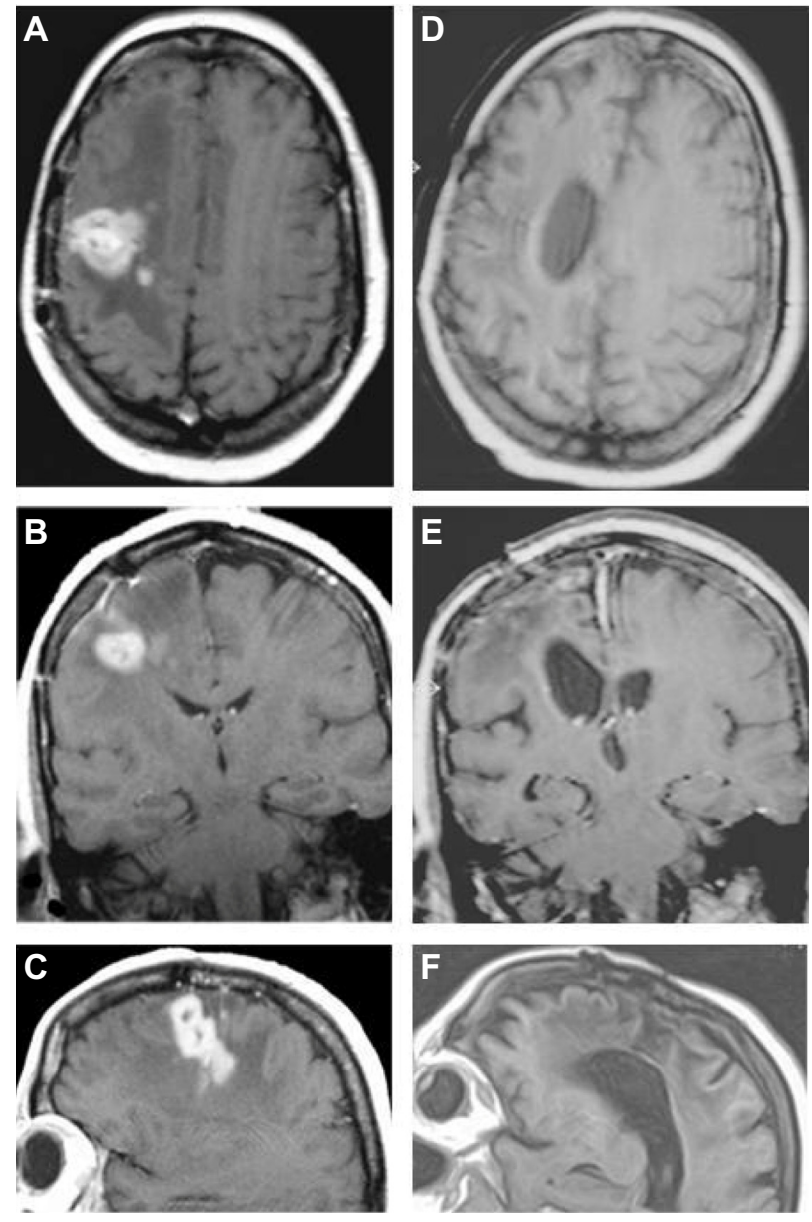

Figure 2 Axial, coronal, and sagittal TI gadolinium enhanced magnetic resonance images before oncolytic HSV treatment (A-C) and at five year follow-up (D-F). Abbreviations: 5yr F/U, 5-year follow-up; HSV, herpes simplex virus. 
the resection site and was felt to represent progression again. CCNU was discontinued, and she enrolled in a study of temozolomide (UAB protocol 9706) and received one course of temozolomide (note that this portion of the patient's care took place before temozolomide use became routine).

Two months after temozolomide treatment, the MRI remained unchanged, representing three consecutive MRI scans showing what was assessed as tumor progression. At that time, she was enrolled into the gene therapy arm of the Neurovir G207 protocol. ${ }^{13}$ Stereotactic injection of $120 \mu \mathrm{L}$ G207 viral suspension containing $1 \times 10^{7}$ plaque-forming units was made into the enhancing region. No tumor biopsy was performed during this initial trial of an oncolytic HSV due to concerns of producing a reparative response to biopsy that could allow the establishment of herpes encephalitis. The patient tolerated the procedure well and postoperative recovery was uneventful. She was discharged with a Karnofsky performance status scale (KPS) score of 90.

A month later, the patient returned for follow-up; MRI showed an increase in the amount of enhancement and so the patient was started on procarbazine therapy of $100 \mathrm{mg} / \mathrm{m}^{2}$, a total dose of $200 \mathrm{mg}$ /day for presumed progression.

Two months later, the patient exhibited new left-sided weakness and her KPS score fell from 70 to 60. A repeat MRI still showed the enhancing lesion, and she underwent craniotomy and debulking of the presumed recurrent tumor. Neuropathologic examination of the resected material identified areas of coagulative necrosis, perivascular inflammation, edema, astrocytosis, and foamy macrophages treatment effect (Figure 3). Residual glioma could not be ruled out due to the appearance of atypical cells; however, our pathologists did not agree as to whether these represented glioma. No mitoses were seen on hematoxylin and eosin examination, but areas of the specimen showed the monoclonal antibody MIB-1 index was $5 \%$, consistent with either residual glioma (down from $33 \%$ at time of original diagnosis) or inflammatory response.

Three months later, the patient's MRI and neurological exam remained unchanged. She was started on an irinotecan (CPT-11) regimen with $125 \mathrm{mg} / \mathrm{m}^{2}$, for a total dose of $250 \mathrm{mg}$ given weekly for 4 weeks followed by a 2-week drug-free holiday. CPT-11 was monitored with complete blood count with differential and platelet count. She received four cycles of CPT-11 but no further treatment.

She remained stable and off of all treatment for nearly 6 years, when she presented with somnolence and altered mental status. MRI revealed a necrotic right frontal mass consistent with recurrence and evidence of impending herniation, for which she underwent right frontal craniotomy with resection of the mass. Postoperative MRI showed a 99\% resection, and pathologic examination of the specimen showed a highly

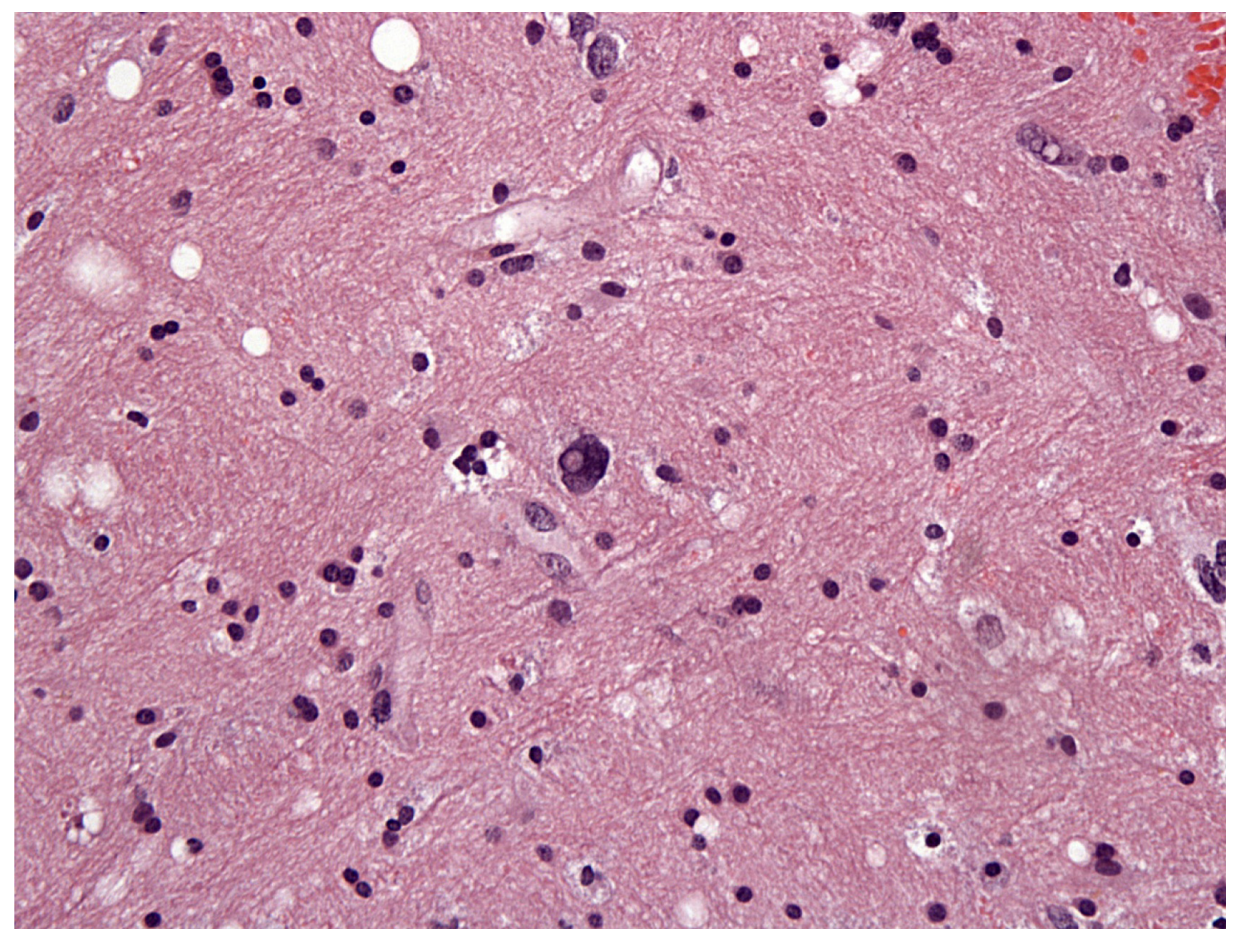

Figure 3 Photomicrograph of hematoxylin and eosin-stained representative tissue from 1998 (post-G207 treatment) resection, demonstrating areas of coagulative necrosis, perivascular inflammation, edema, astrocytosis, and foamy macrophages consistent with treatment effect.

Note: Atypical cells seen could represent inflammation, but tumor is not excluded; $\times 200$. 
cellular astrocytic neoplasm with extensive areas of necrosis and endothelial proliferation. Tumor cells showed marked nuclear pleomorphism with bizarre giant cells and extensive mitosis. Two weeks later, the patient developed a wound infection at the incision site that progressed into intracerebral abscess. She was treated with antibiotics and debridement, and ultimately a ventriculoperitoneal shunt was placed, but due to her infection, she was not eligible for further treatment with G207. No further treatment was given.

Seven months after the resection, she returned to the clinic with symptoms of headache. MRI revealed tumor recurrence involving the entire right hemisphere and compressing the third and fourth ventricles. Surgery was not felt to be a viable option and the patient ultimately succumbed to the disease.

\section{Discussion}

The median survival of patients diagnosed with GBM is about 1 year. ${ }^{17}$ The biology of GBM is unique in that they are locally invasive, rarely metastatic outside the central nervous system, and recur within $2 \mathrm{~cm}$ of the resection cavity in the vast majority of cases. They possess an abnormal and often incomplete blood-brain barrier, making systemic delivery of anticancer drugs unpredictable and erratic. ${ }^{18-20}$ Radiation therapy has met with limited success and is largely limited to external beam therapy. The molecular mechanisms underlying oncogenesis include amplification or mutation of growth factor receptors such as receptor tyrosine kinase-RAS-epidermal growth factor receptor, ${ }^{21}$ loss of tumor suppressor pathways such as p53 and pRB, ${ }^{22}$ and activation of protooncogenes. ${ }^{23}$ Studies that are more recent demonstrate that patients with methylation of the MGMT gene and IDH1 mutations have improved survival. Even patients harboring the most favorable tumors have a 4-year survival rate of approximately $20 \%{ }^{24}$

However, there exists an interesting subpopulation within the GBM patient population labeled as "long-term GBM survivors" or LTGBMS, who survive for more than 3 years after histological diagnosis of GBM. ${ }^{25,26}$ Most LTGBMS are young patients, have KPS scores of 70 or more, and undergo aggressive multimodality therapy. LTGBMS patients are more likely to include patients with MGMT methylation as well as IDH1 mutations, ${ }^{27}$ but may also be an extreme example of a normal distributed variable. As specific molecular mechanisms underlying LTGBMS are determined, it may be possible to induce such mechanisms in all GBM patients by oncolytic virus-mediated and other gene therapy delivery systems.

Oncolytic HSVs are being increasingly utilized in experimental treatment of cancers with some success. ${ }^{28-32}$ HSVs, being naturally neurotropic and possessing a double-stranded DNA genome with a capsid, are amenable to modification with antitumor properties. ${ }^{33} \mathrm{G} 207$ is a genetically engineered HSV with two independent mutations to minimize the risk of wild-type reversion or recombination. Since G207 is a temperate sensitive virus, any development of encephalitis with resultant increase of body temperature greater than $98.6^{\circ} \mathrm{F}$ should prevent viral replication, thereby terminating or reducing adverse effects. In addition, G207 is hypersensitive to acyclovir, the most commonly used antiHSV in the clinic. This is a characteristic which could be exploited in the event encephalitis developed. Oncolysis can also be paired with foreign gene expression such as cytokine production, ${ }^{34}$ chemokine production, and dendritic cell immune induction. ${ }^{35}$ All these factors make HSV an attractive vector for GBM oncolysis.

This is a report of the clinical course and management of a single patient from the initial G207 oncolytic therapy trial who was initially treated with standard conventional therapy of GBM at that time, including biopsy and external beam therapy, followed by brief courses of CCNU and temozolomide. After the viral injection, the ring-enhancing lesion persisted, and the patient was treated briefly with procarbazine, with a presumptive diagnosis of residual/recurrent glioma. In retrospect, this may have been predominantly treatment effect of the G207 injections consistent with a viralmediated pseudoprogression rather than residual glioma as seen on imaging studies. Currently, there exists a paucity of data on imaging changes occurring during and subsequent to oncolytic virus therapy in the brain. ${ }^{36}$

Subsequently, the patient developed hemiparesis and underwent debulking changes interpreted as radiation-induced damage, with areas of edema and foamy macrophage infiltration as well as areas of residual/recurrent glioma versus inflammatory response. Edema and foamy macrophages are also hallmarks of viral-mediated immune response and have been shown in animal models in the brain. ${ }^{37-39}$ Since the HSVmediated postglioma histopathologic treatment effects are not yet established, the presumptive diagnosis of radiation changes may have been incorrect, thus imaging findings may have been partially or completely related to viral treatment.

The patient's course included one cycle of temozolmide prior to resection and four cycles of CPT-11 before receiving no further treatment for the next 6 years, during which she was disease-free. While it is not impossible that the agent responsible for the 6-year remission was CPT-11, it is highly improbable given its poor performance as a single antiglioma agent. ${ }^{40}$ Therefore, it is much more likely that the oncolytic effects of the G207 virus and associated antitumor immune 
responses may have been the mechanism for, or else highly contributed to, the extended tumor-free interval.

Despite our patient's long-standing response to treatment, her tumor did eventually recur. While a second, de novo GBM cannot be ruled out, the location of the tumor at the previous resection site suggests a recurrence of her original GBM. The possibility of the occurrence of an additional mutation that provided a means of escape from immunosurveillance, while conjecture, seems a more plausible explanation.

\section{Conclusion}

In this patient, despite aggressive surgical resection, adjuvant radiotherapy and chemotherapy prior to G207 treatment, tumor progression occurred in typical fashion. However, with G207 oncolytic therapy and brief exposures to additional chemotherapy, the patient in this report experienced an extended survival time of 7.5 years and a 6-year apparently disease-free interval. MGMT status for this patient is unknown, and since temozolomide was not standard treatment during this era, the patient received only a single cycle without evident response. Expected median survival for patients with GBM during this time period was 1 year, and virtually no long-term survivor "tail" was present, making this case even more unusual. A possibly significant finding in this report is the pathologic documentation of edema and foamy macrophage infiltration in the post-G207-treatment specimen, with possible but no indisputable presence of recurrent glioma. Many studies, both preclinical and clinical, have supported the importance of the immune response in producing the effects of oncolytic viral therapy, and certainly this has been demonstrated in G207. ${ }^{34,41}$ Further pathologic studies of glioma patients with possible postoncolytic HSV pseudoprogression will be necessary to determine if the finding in this patient was significant and related to the HSV treatment, or simply a radiation injury response that occurred in the context of oncolytic viral treatment.

This favorable case report suggests that oncolytic GBM therapy may hold promise in the treatment of malignant glioma. However, it is unclear why this particular patient in the G207 study showed favorable remission compared to others in the same cohort. As more patients are treated with oncolytic viruses, a meaningful analysis of molecular changes in those patients with favorable responses may be possible. A better understanding of the mechanism of tumor response to oncolytic $\mathrm{HSV}$, including the contribution of the immune response and subsequent optimization of these parameters, might be exploited to improve the treatment of this relentless disease.

\section{Acknowledgments}

We would like to thank Drs Yancey Gillespie and Cheryl Palmer for input related to the preparation of this manuscript. Funding for this research was provided in part through National Institutes of Health grants P20 CA151129 and P01 CA71933.

\section{Disclosure}

JMM is a cofounder, stockholder, and consultant for Catherex, Inc, as well as a founder and stockholder in Aettis, Inc, which hold intellectual property related to oncolytic HSV. The other authors report no other conflicts of interest in this work.

\section{References}

1. Deorah S, Lynch CF, Sibenaller ZA, Ryken TC. Trends in brain cancer incidence and survival in the United States: Surveillance, Epidemiology, and End Results Program, 1973 to 2001. Neurosurg Focus. 2006;20(4):E1

2. Louis DN. WHO Classification of Tumours of the Central Nervous system, 4th Edition. Lyon: International Agency for Research on Cancer 2007;33-49.

3. Mahaley MS Jr, Mettlin C, Natarajan N, Laws ER Jr, Peace BB. National survey of patterns of care for brain-tumor patients. J Neurosurg. 1989;71(6):826-836.

4. Stupp R, Mason WP, van den Bent MJ, et al; European Organisation for Research and Treatment of Cancer Brain Tumor and Radiotherapy Groups; National Cancer Institute of Canada Clinical Trials Group. Radiotherapy plus concomitant and adjuvant temozolomide for glioblastoma. N Engl J Med. 2005;352(10):987-996.

5. Chang SM, Butowski NA, Sneed PK, Garner IV. Standard treatment and experimental targeted drug therapy for recurrent glioblastoma multiforme. Neurosurg Focus. 2006;20(4):E4.

6. Hegi ME, Diserens AC, Gorlia T, et al. MGMT gene silencing and benefit from temozolomide in glioblastoma. $N \mathrm{Engl} \mathrm{J} \mathrm{Med}$. 2005;352(10):997-1003.

7. Aoki T, Hashimoto N, Matsutani M. Management of glioblastoma Expert Opin Pharmacother. 2007;8(18):3133-3146.

8. Terzis AJA, Niclou SP, Rajcevic U, Danzeisen C, Bjerkvig R. Cell therapies for glioblastoma. Expert Opin Biol Ther. 2006;6(8):739-749.

9. Yu JS, Wheeler CJ, Zeltzer PM, et al. Vaccination of malignant glioma patients with peptide-pulsed dendritic cells elicits systemic cytotoxicity and intracranial T-cell infiltration. Cancer Res 2001;61(3):842-847.

10. Huszthy PC, Goplen D, Thorsen F, et al. Oncolytic herpes simplex virus type-1 therapy in a highly infiltrative animal model of human glioblastoma. Clin Cancer Res. 2008;14(5):1571-1580.

11. Markert JM, Parker JN, Gillespie GY, Whitley RJ. Genetically engineered human herpes simplex virus in the treatment of brain tumours. Herpes. 2001;8(1):17-22.

12. Prados MD, McDermott M, Chang SM, et al. Treatment of progressive or recurrent glioblastoma multiforme in adults with herpes simplex virus thymidine kinase gene vector-producer cells followed by intravenous ganciclovir administration: a phase I/II multi-institutional trial. J Neurooncol. 2003;65(3):269-278.

13. Markert JM, Medlock MD, Rabkin SD, et al. Conditionally replicating herpes simplex virus mutant, G207 for the treatment of malignant glioma: results of a phase I trial. Gene Ther. 2000;7(10):867-874.

14. Todo T, Feigenbaum F, Rabkin SD, et al. Viral shedding and biodistribution of G207, a multimutated, conditionally replicating herpes simplex virus type 1, after intracerebral inoculation in aotus. Mol Ther. 2000;2(6):588-595. 
15. Yazaki T, Manz HJ, Rabkin SD, Martuza RL. Treatment of human malignant meningiomas by G207, a replication-competent multimutated herpes simplex virus 1. Cancer Res. 1995;55(21):4752-4756.

16. Heesters MA, Koudstaal J, Go KG, Molenaar WM. Analysis of proliferation and apoptosis in brain gliomas: prognostic and clinical value. J Neurooncol. 1999;44(3):255-266.

17. Paszat L, Laperriere N, Groome P, Schulze K, Mackillop W, Holowaty E. A population-based study of glioblastoma multiforme. Int $J$ Radiat Oncol Biol Phys. 2001;51(1):100-107.

18. Black KL, Yin D, Konda BM, et al. Different effects of KCa and KATP agonists on brain tumor permeability between syngeneic and allogeneic rat models. Brain Res. 2008;1227:198-206.

19. Wolburg H, Wolburg-Buchholz K, Kraus J, et al. Localization of claudin-3 in tight junctions of the blood-brain barrier is selectively lost during experimental autoimmune encephalomyelitis and human glioblastoma multiforme. Acta Neuropathol. 2003;105(6):586-592.

20. Wu GN, Ford JM, Alger JR. MRI measurement of the uptake and retention of motexafin gadolinium in glioblastoma multiforme and uninvolved normal human brain. J Neurooncol. 2006;77(1):95-103.

21. Layfield LJ, Willmore C, Tripp S, Jones C, Jensen RL. Epidermal growth factor receptor gene amplification and protein expression in glioblastoma multiforme: prognostic significance and relationship to other prognostic factors. Appl Immunohistochem Mol Morphol. 2006;14(1):91-96.

22. Zheng H, Ying H, Yan H, et al. p53 and Pten control neural and glioma stem/progenitor cell renewal and differentiation. Nature. 2008; 455(7216):1129-1133.

23. Cancer Genome Atlas Research Network. Comprehensive genomic characterization defines human glioblastoma genes and core pathways. Nature. 2008;455(7216):1061-1068.

24. Stupp R, Hegi ME, Mason WP, et al; European Organisation for Research and Treatment of Cancer Brain Tumour and Radiation Oncology Groups; National Cancer Institute of Canada Clinical Trials Group. Effects of radiotherapy with concomitant and adjuvant temozolomide versus radiotherapy alone on survival in glioblastoma in a randomised phase III study: 5-year analysis of the EORTC-NCIC trial. Lancet Oncol. 2009;10(5):459-466.

25. Scott JN, Rewcastle NB, Brasher PM, et al. Long-term glioblastoma multiforme survivors: a population-based study. Can J Neurol Sci. 1998;25(3):197-201.

26. Scott JN, Rewcastle NB, Brasher PM, et al. Which glioblastoma multiforme patient will become a long-term survivor? A populationbased study. Ann Neurol. 1999;46(2):183-188.

27. Weller M, Felsberg J, Hartmann C, et al. Molecular predictors of progression-free and overall survival in patients with newly diagnosed glioblastoma: a prospective translational study of the German Glioma Network. J Clin Oncol. 2009;27(34):5743-5750.
28. Benencia F, Courreges MC, Conejo-García JR, et al. Oncolytic HSV exerts direct antiangiogenic activity in ovarian carcinoma. Hum Gene Ther. 2005;16(6):765-778.

29. Derubertis BG, Stiles BM, Bhargava A, et al. Cytokine-secreting herpes viral mutants effectively treat tumor in a murine metastatic colorectal liver model by oncolytic and T-cell-dependent mechanisms. Cancer Gene Ther. 2007;14(6):590-597.

30. Kasuya H, Takeda S, Nomoto S, Nakao A. The potential of oncolytic virus therapy for pancreatic cancer. Cancer Gene Ther. 2005;12(9): 725-736.

31. Pin RH, Reinblatt M, Fong Y. Utilizing alpha-fetoprotein expression to enhance oncolytic viral therapy in hepatocellular carcinoma. Ann Surg. 2004;240(4):659-665; discussion 665-666.

32. Yang CT, Lin YC, Lin CL, et al. Oncolytic herpesvirus with secretable angiostatic proteins in the treatment of human lung cancer cells. Anticancer Res. 2005;25(3B):2049-2054.

33. Shen Y, Nemunaitis J. Herpes simplex virus 1 (HSV-1) for cancer treatment. Cancer Gene Ther. 2006;13(11):975-992.

34. Hellums EK, Markert JM, Parker JN, et al. Increased efficacy of an interleukin-12-secreting herpes simplex virus in a syngeneic intracranial murine glioma model. Neuro Oncol. 2005;7(3):213-224.

35. Benencia F, Courrèges MC, Conejo-García JR, Mohammed-Hadley A, Coukos G. Direct vaccination with tumor cells killed with ICP4-deficient HSVd120 elicits effective antitumor immunity. Cancer Biol Ther. 2006;5(7):867-874.

36. Freeman AI, Zakay-Rones Z, Gomori JM, et al. Phase I/II trial of intravenous NDV-HUJ oncolytic virus in recurrent glioblastoma multiforme. Mol Ther. 2006;13(1):221-228.

37. Eck SL, Alavi JB, Alavi A, et al. Treatment of advanced CNS malignancies with the recombinant adenovirus H5.010RSVTK: a phase I trial. Hum Gene Ther. 1996;7(12):1465-1482.

38. Germano IM, Fable J, Gultekin SH, Silvers A. Adenovirus/herpes simplex-thymidine kinase/ganciclovir complex: preliminary results of a phase I trial in patients with recurrent malignant gliomas. J Neurooncol. 2003;65(3):279-289.

39. Mori I, Goshima F, Koshizuka T, et al. Iba1-expressing microglia respond to herpes simplex virus infection in the mouse trigeminal ganglion. Brain Res Mol Brain Res. 2003;120(1):52-56.

40. Raymond E, Fabbro M, Boige V, et al. Multicentre phase II study and pharmacokinetic analysis of irinotecan in chemotherapy-naïve patients with glioblastoma. Ann Oncol. 2003;14(4):603-614.

41. Markert JM, Liechty PG, Wang W, et al. Phase Ib trial of mutant herpes simplex virus G207 inoculated pre-and post-tumor resection for recurrent GBM. Mol Ther. 2009;17(1):99-207.
Oncolytic Virotherapy

\section{Publish your work in this journal}

Oncolytic Virotherapy is an international, peer-reviewed, open access online journal publishing original research, study protocols, reviews, editorials and commentaries on all aspects of oncolytic virology, namely the application of oncolytic viruses for the treatment of cancer. Specific topics in the journal include: Rationale and theoretical aspects of oncolytic virotherapy including in vitro, in vivo and mathematical

Submit your manuscript here: http://www.dovepress.com/oncolytic-virotherapy-journal

\section{Dovepress}

modeling; and practical application and problem solving in the clinic including identification of potential responders through biomarkers and genetic profiling. The manuscript management system is completely online and includes a very quick and fair peer-review system, which is all easy to use. Visit http://www.dovepress.com/ testimonials.php to read real quotes from published authors. 